\title{
COVID19 seroconversion in an unrelated stem cell donor
}

\author{
Joseph E. Maakaron $\mathbb{1}^{1} \cdot$ David McKenna Jr. ${ }^{1}$. Najla El-Jurdi ${ }^{1}$ Mukta Arora ${ }^{1} \cdot$ Claudio Brunstein $^{1} \cdot$ Steven Devine $^{2}$. \\ Sophia Yohe ${ }^{1} \cdot$ Daniel Weisdorf $\mathbb{C}^{1}$
}

Received: 27 August 2020 / Revised: 9 September 2020 / Accepted: 16 September 2020 / Published online: 22 September 2020

(c) The Author(s), under exclusive licence to Springer Nature Limited 2020

\section{To the Editor:}

The COVID19 pandemic requires no introduction anymore with a total of 12.5 million cases reported worldwide and more than 500,000 deaths as of July 30, 2020 [1]. Of particular interest to the audience of this journal is the impact of SARS-CoV2 infection in immunosuppressed patients and patients with hematological malignancies. The COVID19 and Cancer Consortium database (CCC19) is an ongoing clinical trial investigating the impact of SARS-CoV2 infection in patients with malignancies. They have reported an increased mortality (13\%) among 1000 patients with different cancer types [2]. Hematopoietic stem cell transplant (HCT) programs have all adapted and gradually adopted workflow modifications to mitigate infectious risk on both patients and staff. The National Marrow Donor Program (NMDP) has also adjusted its workflow by screening donors of HCT prior to collection of hematopoietic stem cells (HSC) followed by cryopreservation of the graft. Most transplant centers have also adopted a policy of not initiating conditioning regimens prior to receipt of the cryopreserved HSCs given the possibility of delays in air travel and shipping [3].

We herein report the first occurrence of an unrelated HSC donor who tested positive for SARS-CoV2 shortly after donating HSCs, our approach to management, and short-term follow-up. We review the literature about transmission of SARS-CoV2 in blood and end with a brief set of recommendations about lessons learned.

Joseph E. Maakaron

maaka001@umn.edu

1 Department of Medicine, Division of Hematology, Oncology and Transplantation, University of Minnesota, Minneapolis, MN, USA

2 National Marrow Donor Program, Minneapolis, MN, USA

\section{Case}

Our index patient is a 53-year-old woman with therapy-related acute myeloid leukemia with a TP53 mutation. She was in complete remission but had evidence of minimal residual disease by next generation sequencing (NGS). She was set to receive a total-body irradiation-based, myeloablative transplant from a HLA-matched donor (11/12; DP permissive mismatch), who was an ABO major mismatch. The donor was tested negative for SARS-CoV2 $48 \mathrm{~h}$ prior to undergoing HSC harvest from the bone marrow per what is currently standard of practice of the donor center. Two days following the harvest, he reported symptoms of a sore throat and fatigue. He also reported that one of the members of his household was diagnosed with COVID19. After the close contact tested positive, the donor was retested for SARS-CoV2 and found to be positive. He displayed mild symptoms that spontaneously resolved. At the time of confirmed positive testing, the patient had completed $80 \%$ of her scheduled TBI and the decision was made to move forward with the transplant given the myeloablative conditioning already administered. Highefficiency particulate-air (HEPA) filtration was activated in the patient's room. A segment from the bone marrow product, cryopreserved at $-80^{\circ} \mathrm{C}$ was tested for SARS-CoV2 using qRT-PCR to viral targets N1 and N2 (as per the CDC 2019 Novel Coronavirus (2019-nCoV) Real-Time Reverse Transcriptase (RT)-PCR Diagnostic Panel) and was negative [4]. Given the negative result on the bone marrow product and the lack of evidence for blood transmission of coronavirus, we used standard precautions and did not isolate the patient. The patient did not display any infectious signs or symptoms during the first 14 days of her post-transplant course and is currently recovering almost one month from her transplant.

\section{Discussion}

The medical community responded in a swift and nimble manner to the COVID19 pandemic by instituting policies 
to minimize both staff and patient exposure and to account for the logistic barriers that have arisen. The NMDP was among those early responders when they rallied to get flight exemptions for couriers carrying stem cell products, rapidly analyzed and the CIBMTR made available data regarding the safety of cryopreservation in different disease states, instituted policies and communicated with transplant centers about the procurement and transportation of stem cell products. Donors were routinely screened with the questionnaire. Once testing became widely available, it was added, whenever possible, prior to donation to ensure utmost safety. Our transplant center, along with others, instituted policies where conditioning of the patient is delayed until the product has safely arrived.

Despite all these measures, new challenges continue to arise despite our best efforts to predict and mitigate them. We herein presented a patient who was set to receive a product that potentially could have been contaminated with SARS-CoV2. When we learned of the donor testing, it would have been difficult to delay the transplant. After discussing the risks and benefits of proceeding versus aborting the transplant, we proceeded as scheduled.

SARS-CoV2 ribonucleic acid (RNA) has been detected in the blood of patients with SARS-CoV2 [5-7]. However, there is no reported evidence that SARS-CoV2 is transmissible in the blood. In fact, there is evidence that it is unlikely to be transmitted. A report of 18 patients with SARS-CoV2 infection and varying levels of symptoms examined the rate of positivity of RNA qRT-PCR in whole blood, serum, and plasma. Testing was repeated between 1 and 8 times on each individual patient for a total of 77 samples. Only one sample had weak positivity (179 copies/ml) [8]. The mere detection of RNA in the blood of donors does not imply transmissibility of the infectious virus itself. In another study examining SARS-CoV2 virology, RNA was consistently recovered from stool, but no viral particles were isolated in cultures, highlighting the discrepancy between RNA detection and infectivity [9]. In fact, respiratory viruses are generally not considered to be transmissible by blood. The two most commonly cited examples are Severe Acute Respiratory Syndrome (SARS) virus and the Middle East Respiratory Syndrome (MERS) coronavirus that have caused epidemics in recent history but have not been reported to be transported by blood products [10]. There is also no evidence that respiratory viruses, including SARS-CoV2, survive cryopreservation or other pathogen-reduction methods. Reagents currently in use, such as the Mirasol Pathogen Reduction Technology, have been shown to be able to inactivate the virus in blood products [11]. The clinical syndrome of COVID19 happens after the virus infects respiratory epithelial cells and
Donor center

Vigilant screening with targeted questionnaires.

- Serological and nucleic acid testing for SARS-CoV2 if available

- Attention to signs and symptoms including presence of lymphopenia, anosmia, elevated LDH

- Follow up with donors within 7 days after donating

- Expeditious data reporting

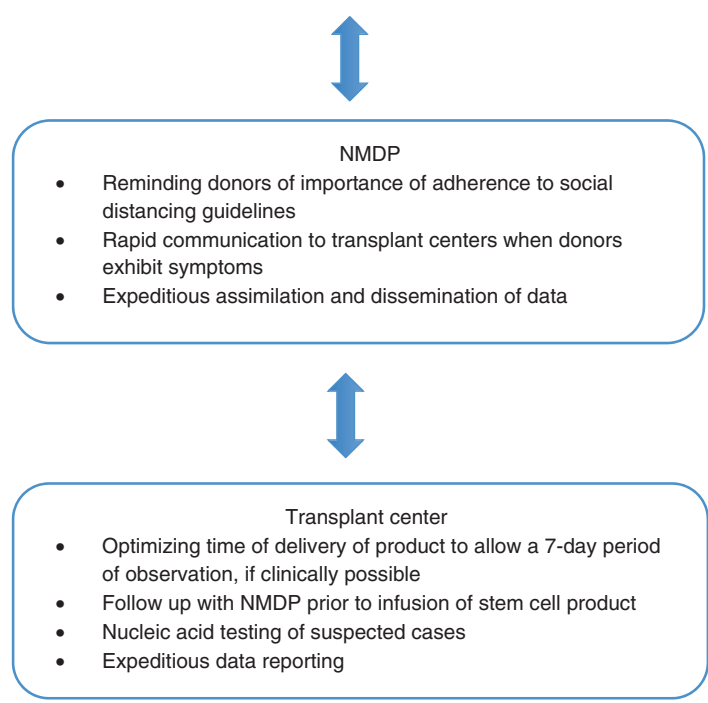

Fig. 1 Interplay between three centers involved in procurement and delivery of stem cell product.

replicates within them. There is no evidence that this syndrome can occur if live viral particles are circulating in blood. In one report from South Korea, seven blood and blood product donors developed COVID19 after donating. Their donations resulted in a total of 17 products that were transfused without any evidence of a clinical syndrome occurring in the recipients and subsequent viral PCR testing of the product segments were found to be negative [12]. In summary, there is no clear evidence to suggest transmission of SARS-CoV2 by blood and blood products.

Coordinating stem cell donation, travel and delivery, and timing of transplant in a patient with aggressive malignancies in a pandemic is challenging and unexpected situations will continue to arise. However, donor centers and the NMDP must continue to exercise vigilance in screening donors and thoroughly investigating recent exposures to SARS-CoV2, including non-adherence to social distancing guidelines. We present our recommendations in Fig. 1.

\section{Compliance with ethical standards}

Conflict of interest The authors declare that they have no conflict of interest.

Publisher's note Springer Nature remains neutral with regard to jurisdictional claims in published maps and institutional affiliations. 


\section{References}

1. Coronavirus Disease (COVID-19) Situation Reports. https://www. who.int/emergencies/diseases/novel-coronavirus-2019/situationreports (2020).

2. Kuderer NM, Choueiri TK, Shah DP, Shyr Y, Rubinstein SM, Rivera DR, et al. Clinical impact of COVID-19 on patients with cancer (CCC19): a cohort study. Lancet. 2020;395:1907-18.

3. Updates for Transplant Centers and Cooperative Registries. https://network.bethematchclinical.org/news/nmdp/be-the-matchresponse-to-covid-19/updates-for-transplant-centers-and-coopera tive-registries/ (2020).

4. CDC. Information for Laboratories about Coronavirus (COVID19). Cent. Dis. Control Prev. 2020. https://www.cdc.gov/corona virus/2019-ncov/lab/virus-requests.html (2020).

5. Kim JY, Ko J-H, Kim Y, Kim Y-J, Kim J-M, Chung Y-S, et al. Viral load kinetics of SARS-CoV-2 infection in first two patients in Korea. $J$ Korean Med Sci. 2019;35. https://doi.org/10.3346/jkms.2020.35.e86.

6. Huang C, Wang Y, Li X, Ren L, Zhao J, Hu Y, et al. Clinical features of patients infected with 2019 novel coronavirus in Wuhan, China. Lancet. 2020;395:497-506.
7. Zhang W, Du R-H, Li B, Zheng X-S, Yang X-L, Hu B, et al. Molecular and serological investigation of 2019-nCoV infected patients: implication of multiple shedding routes. Emerg Microbes Infect. 2020;9:386-9.

8. Corman VM, Rabenau HF, Adams O, Oberle D, Funk MB, KellerStanislawski B, et al. SARS-CoV-2 asymptomatic and symptomatic patients and risk for transfusion transmission. Transfus (Paris). 2020; 60:1119-22.

9. Wölfel R, Corman VM, Guggemos W, Seilmaier M, Zange S, Müller MA, et al. Virological assessment of hospitalized patients with COVID-2019. Nature. 2020;581:465-9.

10. Katz LM. Is SARS-CoV-2 transfusion transmitted? Transfus (Paris). 2020;60:1111-4.

11. Keil SD, Ragan I, Yonemura S, Hartson L, Dart NK, Bowen R. Inactivation of severe acute respiratory syndrome coronavirus 2 in plasma and platelet products using a riboflavin and ultraviolet light-based photochemical treatment. Vox Sang. 2020. https://doi. org/10.1111/vox.12937.

12. Kwon S, Kim E, Jung YS, Jang JS, Cho N Post-donation COVID19 identification in blood donors. Vox Sang. 2020. 